\section{Medical management of glaucoma}

James Tsai and Max Forbes

Professional Communications, Inc., (approx £15.50) (May be ordered by telephone: +1 800337 9838.)

Oklahoma, USA, 2003, 240 pp

ISBN 1-884735-80-0

Eye (2005) 19, 726.

doi:10.1038/sj.eye. 6701552

This pocket-sized book is easily portable and could easily fit in a white coat for those of us who still wear one. It has obviously been written with the aim of providing a clear introduction to the aetiology, pathology, and management of glaucoma. Despite its size, it is full of useful information. The contents are clearly laid out with page markers for easy access to particular chapters.
The book has been well written and is easy to read. Each section also contains key references for further reading.

As the title suggests, the theme is medical and not surgical, although management of trabeculectomy complications is covered briefly. There are useful sections on the recent major glaucoma studies and their implications. The pharmacological characteristics of all the main glaucoma agents including the new prostaglandin analogues are described and compared.

Examination of the glaucoma patient and visual field interpretation are all explained in easily understandable terms.

The book has been written from an American point of view, so some of the treatment algorithms may differ from UK practice guidelines.
There are only a few basic illustrations in the text with no clinical photographs, but charts and tables are used well to clearly illustrate the authors' points.

Having said that, for the price, I cannot rate this book highly enough. It contains more information than many more expensive texts. I would recommend this book to all trainee ophthalmologists as well as consultants who want a concise summary of recent developments in the diagnosis and management of glaucoma. As far as I am aware, the book is available to order on-line only at present.

A Inglis

Royal Hallamshire Hospital Glossop Road Sheffield S10 2JF, UK 